\title{
Leader-Follower Fixed-Time Group Consensus Control of Multiagent Systems under Directed Topology
}

\author{
Yilun Shang and Yamei Ye \\ School of Mathematical Sciences, Tongji University, Shanghai 200092, China \\ Correspondence should be addressed to Yilun Shang; shylmath@hotmail.com
}

Received 19 January 2017; Accepted 1 March 2017; Published 16 March 2017

Academic Editor: Jinde Cao

Copyright (c) 2017 Yilun Shang and Yamei Ye. This is an open access article distributed under the Creative Commons Attribution License, which permits unrestricted use, distribution, and reproduction in any medium, provided the original work is properly cited.

This paper investigates the fixed-time group consensus problem for a leader-follower network of integrators with directed topology. A nonlinear distributed control protocol, based on local information, is proposed such that the follower agents in each subgroup are able to track their corresponding leaders in a prescribed convergence time regardless of the initial conditions. Simulation examples are presented to demonstrate the availability of our theoretical results.

\section{Introduction}

The past decade has witnessed an increasing attention on cooperative control of multiagent systems due to its broad applications in diverse fields including distributed computation, formation of unmanned vehicles, data fusion of sensor networks, synchronization of coupled chaotic oscillators, flocking, and swarming. A fundamental issue in coordination of multiagent systems is the consensus problem, which requires that all the agents should reach an agreement using the local information of their neighbors, which is determined by the underlying communication topology. Toward this aim, an essential step is to design proper distributed control laws such that as time goes on, all the agents converge to a consistent value asymptotically; see, e.g., [1-3] for some representative works on this topic. More backgrounds and applications of consensus problems can be found in the survey papers $[4,5]$ and references therein.

Most of the existing work focuses on complete consensus in the sense that all agents ultimately achieve a common group decision value [4]. However, in many real-world circumstances in cooperative control, a group of agents should be able to split into multiple subgroups and different consistent states are desired with the changes of environments, situations, and tasks. Examples include pattern formation of bacteria colonies, predator evasion and separated foraging for animal herds, cooperative task searching for autonomous vehicles, and coordinated military operations. As a generalization of complete consensus problems, group consensus has been studied intensively in recent years [611], where the agents in a network are divided into multiple subgroups and the states of agents in each subgroup can reach an individual consistent state asymptotically. For example, a group consensus protocol is proposed in [6] for the firstorder continuous-time multiagent systems with switching topology by using double-tree-form transformation, which converts the group consensus problem into the stability of a corresponding reduced system. A combinatorial necessary and sufficient condition for discrete-time group consensus problem is proposed in [11]. Moreover, many applications in cooperative control require dynamic leaders, which can be virtual for their followers $[1,12]$. The leader-follower consensus problems are more challenging than their leaderless counterparts because these controllers not only ensure the consensus of the followers but also determine the final consensus value, which is defined by the dynamic leader. Recently, a leader-follower group consensus protocol has been proposed for second-order multiagent systems in [13]. An event-triggered control protocol has been introduced in [14] to achieve leader-follower group consensus for nonlinear multiagent systems.

It is worth noting that, for the aforementioned works, the (group) consensus can only be reached in an asymptotic manner; namely, the settling time is infinite. In many 
applications, a high speed convergence characterized by a finite-time control law is essential. Finite-time consensus can lead to better disturbance rejection and more robustness against uncertainties [15]. Numerous finite-time controllers have been designed for both complete consensus problems (e.g., [16-20]) and group consensus problems (e.g., [21, 22]). Recently, a new concept, called fixed-time stability, has been proposed in [23], which guarantees a finite settling time independent of initial conditions. This fixed-time approach is promising since the knowledge of initial conditions is usually not available in advance in distributed systems. Based on the fixed-time stability notion, many new results are reported. For example, in the leaderless cases, the fixed-time average consensus has been investigated in [24-26] for the first-order integrator systems over undirected topologies. Several fixedtime group consensus protocols are analyzed in [27] over undirected topologies. Fixed-time leader-follower consensus for second-order multiagent systems with bounded input uncertainties is studied in [28], while unknown nonlinear inherent dynamics are considered in [29] for first-order leader-follower multiagent systems. The fixed-time masterslave synchronization control problems for the delayed Cohen-Grossberg neural networks and delayed memristorbased recurrent neural networks are studied in [30, 31], respectively. Two fixed-time distributed control strategies are proposed in [32] such that leaderless and leader-follower consensus can be reached over directed communication topology. It is worth noting that previous works heavily rely on the symmetry property of the network topology, and [32] is the only one dealing with general asymmetric topology concerning fixed-time consensus problems to our knowledge.

In this paper, we aim to move a further step along this line of research by studying leader-follower fixed-time group consensus for multiagent systems under directed topology. The contribution of this paper is twofold. First, a generalization of the fixed-time leader-follower consensus protocol is proposed to accommodate both directed topology and multiple leaders. Second, an explicit estimation of the settling time is presented regardless of initial conditions.

The rest of the paper is organized as follows. Section 2 provides some preliminaries and formulates the leaderfollower group consensus problem. Section 3 is devoted to the analysis of our proposed fixed-time control strategy. Some numerical examples are given in Section 4 to illustrate the correctness of the obtained theoretical results. A conclusion is drawn in Section 5.

\section{Background and Preliminaries}

To start with, we fix some standard notations that will be used throughout the paper. The cardinality of a set $S$ and the absolute value of a number $s \in \mathbb{R}$ are denoted by $|S|$ and $|s|$, respectively. Let $\mathbb{R}_{+}$represent the set of nonnegative real numbers. Denote by $M^{\mathrm{T}}$ the transpose of a matrix $M$. The maximum and minimum eigenvalues of a symmetric matrix $M$ are denoted by $\lambda_{\max }(M)$ and $\lambda_{\min }(M)$, respectively. $1_{N} \in \mathbb{R}^{N}$ is a vector with all the entries being 1 , and $\operatorname{diag}\left(a_{1}, \ldots, a_{N}\right) \in \mathbb{R}^{N \times N}$ is a diagonal matrix with diagonal entries $a_{1}, \ldots, a_{N}$. Similar notations will be adopted for block diagonal matrices. For a vector $x=\left(x_{1}, \ldots, x_{N}\right)^{\mathrm{T}} \in \mathbb{R}^{N}$ and $a \geq 0$, we define $\left\lfloor\left. x\right|^{a}=\left(\operatorname{sgn}\left(x_{1}\right)\left|x_{1}\right|^{a}, \ldots, \operatorname{sgn}\left(x_{N}\right)\left|x_{N}\right|^{a}\right)^{\mathrm{T}}\right.$, where $\operatorname{sgn}(\cdot)$ is the signum function. For $p>0$, the $p$-norm $\|\cdot\|_{p}$ is defined as $\|x\|_{p}=\left(\sum_{i=1}^{N}\left|x_{i}\right|^{p}\right)^{1 / p}$ for a vector $x \in \mathbb{R}^{N}$. The following lemma relating different norms is often used in the analysis of fixed-time consensus problems, a proof of which can be found in [33].

Lemma 1. Let $x \in \mathbb{R}^{N}$ and $p>q>0$. Then

$$
\|x\|_{p} \leq\|x\|_{q} \leq N^{1 / q-1 / p}\|x\|_{p} .
$$

2.1. Graph Theory. The communication network of a multiagent system can often be described by a directed graph [34]. Let $G=(V, E, A)$ be a weighted directed graph, where the node set $V=\{1,2, \ldots, N\}$ represents $N$ follower agents and the edge set $E \subseteq V \times V$ describes the information exchange among the followers. Here, $A=\left(a_{i j}\right) \in \mathbb{R}^{N \times N}$ is the associated weighted adjacency matrix of the graph, where $a_{j i} \neq 0$ if $(i, j) \in E$ and $a_{j i}=0$ otherwise. A path in $G$ from $i_{1}$ to $i_{k}$ is a sequence of distinct nodes $\left(i_{1}, \ldots, i_{k}\right)$ such that $\left(i_{j}, i_{j+1}\right) \in E$ for $j=1, \ldots, k-1 . G$ is said to have a spanning tree if there exists a node, called root, which has a path to any other nodes in the graph $G$. The (in-degree) Laplacian matrix of $G$ is defined as $L=\left(l_{i j}\right) \in \mathbb{R}^{N \times N}$ with $l_{i i}=\sum_{j \neq i} a_{i j}$ and $l_{i j}=-a_{i j}$ for $i \neq j$. It is well-known that $L$ is positive semidefinite and zero is an eigenvalue of $L$ with the eigenvector $1_{N}$. Moreover, if $G$ contains a spanning tree, then zero is algebraically simple and all other eigenvalues of $L$ are with positive real parts [3]. Let $\bar{G}$ be the directed graph with node set $V \cup\{0\}$ and edge set having the form $E \cup\{(0, i) \mid$ for some $i \in V\}$. Assume that $B=\operatorname{diag}\left(b_{1}, \ldots, b_{N}\right)$ is a nonnegative diagonal matrix with $b_{i}>0$ if and only if $(0, i)$ is an edge in $\bar{G}$. Some properties of $H:=L+B$ are characterized by the following lemma.

Lemma 2 (see [35]). If $\bar{G}$ has a spanning tree with 0 being the root, then $\mathrm{H}$ is nonsingular. Let

$$
\begin{aligned}
\left(p_{1}, \ldots, p_{N}\right)^{T} & =H^{-T} 1_{N}, \\
P & =\operatorname{diag}\left(p_{1}, \ldots, p_{N}\right), \\
Q & =P H+H^{T} P .
\end{aligned}
$$

Then both matrices $P$ and $Q$ are positive definite.

To explore the group consensus, a grouping $\mathscr{G}=\left\{\mathscr{G}_{1}, \ldots\right.$, $\left.\mathscr{G}_{K}\right\}$ of the graph $G$ is defined by dividing its node set into disjoint subgroups $\left\{\mathscr{G}_{k}\right\}_{k=1}^{K}$. In other words, $\mathscr{G}$ satisfies $\bigcup_{k=1}^{K} \mathscr{G}_{k}=V$ and $\mathscr{G}_{k} \cap \mathscr{G}_{k^{\prime}}=\emptyset$ for $k \neq k^{\prime}$. We write $\mathscr{G}_{1}=\left\{1, \ldots, r_{1}\right\}, \mathscr{G}_{2}=\left\{r_{1}+1, \ldots, r_{2}\right\}, \ldots, \mathscr{G}_{K}=\left\{r_{K-1}+\right.$ $1, \ldots, N\}$ and let $r_{0}=0$ and $r_{K}=N$. We assume that the interactions between agents in the same subgroup are cooperative; namely, $a_{i j} \geq 0$ if $i, j \in \mathscr{G}_{k}$ for some $k$. Each subgroup $\mathscr{G}_{k}(1 \leq k \leq K)$ inherits the structure of $G$ naturally in the sense of induced subgraph [34]. For each $1 \leq k \leq K$, the (in-degree) Laplacian matrix of $\mathscr{G}_{k}$ is similarly defined as $L_{k}=\left(l_{i j}\right) \in \mathbb{R}^{\left|\mathscr{G}_{k}\right| \times\left|\mathscr{G}_{k}\right|}$ with $l_{i i}=\sum_{j \in \mathscr{G}_{k}, j \neq i} a_{i j}$ and $l_{i j}=-a_{i j}$ for $i \neq j$. 
Let $\Theta=\left\{\theta_{1}, \theta_{2}, \ldots, \theta_{K}\right\}$ be the set of $K$ virtual leader agents. We make the following assumption.

Assumption 3. Fix any $k \in\{1, \ldots, K\}$. For each $i \in \mathscr{G}_{k}$, there is a path from $\theta_{k}$ to $i$. Moreover, there is no edge from $\theta_{k}$ to $j \in \mathscr{G}_{k^{\prime}}$ for $k^{\prime} \neq k$.

Assumption 3 indicates that the follower agents in each subgroup $\mathscr{G}_{k}$ have access to the information of their own leader $\theta_{k}$ but not to that of the other leaders directly. This assumption eases analysis for multiple leaders, which is also adopted in $[13,14]$. We assume that the weight $a_{i \theta_{k}} \geq 0$ for $i \in \mathscr{G}_{k}$ and denote $B_{k}=\operatorname{diag}\left(a_{r_{k-1}+1, \theta_{k}}, \ldots, a_{r_{k} \theta_{k}}\right) \in \mathbb{R}^{\left|\mathscr{G}_{k}\right| x\left|\mathscr{S}_{k}\right|}$ for $1 \leq k \leq K$. Note that, under Assumption 3 , the matrix $H_{k}:=L_{k}+B_{k}$ is nonsingular by Lemma 2 . Moreover,

$$
\begin{aligned}
P_{k} & =\operatorname{diag}\left(p_{k, r_{k-1}+1}, \ldots, p_{k, r_{k}}\right), \\
Q_{k} & =P_{k} H_{k}+H_{k}^{\mathrm{T}} P_{k}
\end{aligned}
$$

are positive definite, where $\left(p_{k, r_{k-1}+1}, \ldots, p_{k, r_{k}}\right)^{\mathrm{T}}=H_{k}^{-\mathrm{T}} 1_{\left|\mathscr{S}_{k}\right|}$. Define the block diagonal matrix $Q$ by $Q=\operatorname{diag}\left(Q_{1}, \ldots, Q_{K}\right) \epsilon$ $\mathbb{R}^{N \times N}$, which is also positive definite.

Assumption 4. Fix any $k \in\{1, \ldots, K\}$. For each $i \in \mathscr{G}_{k}$ and $k^{\prime} \neq k, \sum_{j \in \mathscr{G}_{k^{\prime}}} a_{i j}=0$. Moreover, for each $i \in \mathscr{G}_{k}$, $\sum_{k^{\prime} \neq k} \sum_{j \in \mathscr{G}_{k^{\prime}}}\left|a_{i j}\right| \leq a_{i \theta_{k}}$. These inequalities hold strictly for at least one $i \in V$.

The first part of Assumption 4, commonly known as the intergroup balance condition, indicates a balance of influence between an agent in a subgroup and all agents in any other subgroup and is typically required for group consensus problems in the existing literature; see, e.g., [6-11, 13, 14, 27, 36]. The second part of Assumption 4 suggests that, for each follower agent $i$, the accumulated influence stemming from agents in other subgroups should be dominated by that from the agent's leader. In bird flight formation, for example, it has been observed that the flock leaders often have strong influence while the groups of followers are evolved to interact with proximal 6-7 birds only with minimal information exchange rate $[37,38]$. This assumption plays a key role in our analysis of fixed-time consensus over directed network topology.

2.2. Fixed-Time Stability. Consider the general differential equation

$$
\begin{aligned}
& \dot{x}(t)=f(t, x(t)), \\
& x(0)=x_{0},
\end{aligned}
$$

where $x \in \mathbb{R}^{n}$ and $f: \mathbb{R}_{+} \times \mathbb{R}^{n} \rightarrow \mathbb{R}^{n}$ is a nonlinear function. The solutions of (4) are understood in the sense of Filippov [39]. Suppose that the origin is an equilibrium of (4).

Definition 5 (see [15]). The origin of system (4) is a globally finite-time equilibrium if there is a function $T: \mathbb{R}^{n} \rightarrow \mathbb{R}_{+}$, called settling time function, such that, for all $x_{0} \in \mathbb{R}^{n}$, the solution $x\left(t, x_{0}\right)$ of system (4) is defined and $x\left(t, x_{0}\right) \in \mathbb{R}^{n}$ for $t \in\left[0, T\left(x_{0}\right)\right)$ and $\lim _{t \rightarrow T\left(x_{0}\right)} x\left(t, x_{0}\right)=0$.

Definition 6 (see [23]). The origin of system (4) is said to be globally fixed-time stable if it is globally finite-time stable and the settling time function $T\left(x_{0}\right)$ is bounded; namely, there is some $T_{\max }>0$ such that $T\left(x_{0}\right) \leq T_{\max }$ for any $x_{0} \in \mathbb{R}^{n}$.

For example, the origin of the simple scalar system $\dot{x}=$ $-x^{1 / 3}$ is a globally finite-time equilibrium with $T\left(x_{0}\right)=$ $(3 / 2) \sqrt[3]{\left|x_{0}\right|^{2}}$. The origin of $\dot{x}=-\lfloor x\rceil^{1 / 3}-\lfloor x\rceil^{2}$ is a globally fixed-time equilibrium because $T\left(x_{0}\right) \leq \pi$ for any $x_{0} \in \mathbb{R}$.

Lemma 7 (see $[24,32])$. Suppose that there is a continuous radially unbounded function $\mathrm{V}: \mathbb{R}^{n} \rightarrow \mathbb{R}_{+}$such that (i) $\mathrm{V}(x)=0 \Leftrightarrow x=0$ and (ii) any solution $x(t)$ of (4) satisfies the inequality $\dot{\mathrm{V}}(x(t)) \leq-a \mathrm{~V}^{\mathrm{P}}(x(t))-b \mathrm{~V}^{q}(x(t))$ for some $a, b>0$, $p=1-1 /(2 \mu), q=1+1 /(2 \mu)$, and $\mu>1 / 2$. Then the origin is globally fixed-time stable and the following estimate of the settling time holds:

$$
T\left(x_{0}\right) \leq T_{\max }=\frac{\pi \mu}{\sqrt{a b}}, \quad \forall x_{0} \in \mathbb{R}^{n} .
$$

This lemma provides a good estimate of the settling time independent of the initial conditions.

2.3. Problem Formulation. We consider the following multiagent system with $N$ follower agents and $K$ virtual leaders governed by

$$
\begin{aligned}
\dot{x}_{i} & =u_{i}, \quad i \in V, \\
\dot{x}_{\theta_{k}} & =u_{\theta_{k}}, \quad k \in\{1, \ldots, K\},
\end{aligned}
$$

where $x_{i} \in \mathbb{R}^{m}$ (resp., $x_{\theta_{k}} \in \mathbb{R}^{m}$ ) is the state of agent $i$ (resp., leader $\theta_{k}$ ) and $u_{i} \in \mathbb{R}^{m}$ (resp., $u_{\theta_{k}} \in \mathbb{R}^{m}$ ) is the control input of agent $i$ (resp., leader $\theta_{k}$ ). The unknown input $u_{\theta_{k}}$ is assumed to be bounded by a known constant $\omega$; that is, $\left\|u_{\theta_{k}}\right\|_{\infty} \leq \omega$ for all $1 \leq k \leq K$.

In this paper, we extend the notion of fixed-time equilibrium in Definition 6 to the setting of coordination control of multiagent systems and define the leader-follower fixed-time group consensus as follows.

Definition 8. The multiagent system (6) is said to achieve leader-follower fixed-time group consensus if, for some appropriately designed distributed inputs $\left\{u_{i}\right\}_{i \in V}$, there exists a constant $T_{\max }>0$ so that, for any initial condition of the agents and the bounded inputs $\left\{u_{\theta_{k}}\right\}_{k=1}^{K}$, it holds that $x_{i}(t)=$ $x_{\theta_{k}}(t)$ for all $i \in \mathscr{G}_{k}, k=1, \ldots, K$, and $t \geq T_{\max }$.

For simplicity, we assume $m=1$ in the following. However, the analysis is valid for $m>1$ by using the properties of the Kronecker product. 


\section{Fixed-Time Group Consensus Analysis under Directed Topology}

In this section, motivated by the fixed-time control techniques used in $[24,27,32]$, we design the following distributed controller for follower agents in order to realize leaderfollower fixed-time group consensus:

$$
\begin{aligned}
u_{i}= & \alpha\left\lfloor\sum_{j \in \mathscr{G}_{k} \cup \theta_{k}} a_{i j}\left(x_{j}-x_{i}\right)+\sum_{k^{\prime} \neq k} \sum_{j \in \mathscr{G}_{k^{\prime}}} a_{i j} x_{j}\right]^{p} \\
& +\beta\left\lfloor\sum_{j \in \mathscr{G}_{k} \cup \theta_{k}} a_{i j}\left(x_{j}-x_{i}\right)+\sum_{k^{\prime} \neq k} \sum_{j \in \mathscr{G}_{k^{\prime}}} a_{i j} x_{j}\right]^{q} \\
& +\gamma \operatorname{sgn}\left(\sum_{j \in \mathscr{G}_{k} \cup \theta_{k}} a_{i j}\left(x_{j}-x_{i}\right)+\sum_{k^{\prime} \neq k} \sum_{j \in \mathscr{G}_{k^{\prime}}} a_{i j} x_{j}\right),
\end{aligned}
$$

for $i \in \mathscr{G}_{k}, k=1, \ldots, K$, where $\alpha, \beta>0, \gamma \geq \omega$ are positive control gains, $p \in(0,1)$, and $q=1 / p$. Note that only local information between neighboring agents is needed.

Assumption 9. Denote $\rho=\max _{1 \leq k \leq K, i \in \mathscr{G}_{k}} \sum_{k^{\prime} \neq k} \sum_{j \in \mathscr{G}_{k^{\prime}}} p_{k i}\left|a_{i j}\right|$, where $\left\{p_{k i}\right\}$ are defined in (3). Assume that $\lambda_{\text {min }}(Q)>\rho$.

Note that Assumption 9 is feasible since $\rho$ will tend to zero when $\left|a_{i j}\right|$ tends to zero for $i \in \mathscr{G}_{k}, j \in \mathscr{G}_{k^{\prime}}$, and $k^{\prime} \neq k$. On the other hand, $Q$ is a positive definite matrix, which does not rely on these intergroup weights $\left\{a_{i j}\right\}$. Assumption 9, together with Assumption 4, implies intuitively that the coupling strengths within each subgroup and among the leader and its followers are strong enough while the coupling strengths between different subgroups should be weak. Assumptions 3, 4 , and 9 are the usual standard ones when considering the problems of group consensus or cluster synchronization.

Theorem 10. Under Assumptions 3, 4, and 9, the multiagent system (6) with protocol (7) achieves leader-follower fixed-time group consensus, and the convergence time is bounded by

$$
T_{\max }=\frac{c_{1} \pi(1+p)}{c_{2}\left(\lambda_{\min }(Q)-\rho\right)(1-p)},
$$

where

$$
\begin{aligned}
c_{1}= & \left(\frac{\bar{p} \alpha}{p+1}\right)^{2 p /(p+1)}+\left(\frac{\bar{p} \beta}{q+1}\right)^{2 p /(p+1)} \\
& +2^{(q-1) /(q+1)}\left(\frac{\bar{p} \alpha}{p+1}\right)^{2 q /(q+1)} \\
& +2^{(q-1) /(q+1)}\left(\frac{\bar{p} \beta}{q+1}\right)^{2 q /(q+1)}
\end{aligned}
$$

$$
c_{2}= \begin{cases}\min \left\{\beta^{2} N^{1-2 q}, \alpha^{2}\right\}, & \text { if } 0<p \leq \frac{1}{2} ; \\ \min \left\{\beta^{2} N^{1-2 q}, \alpha^{2} N^{1-2 p}\right\}, & \text { if } \frac{1}{2} \leq p<1,\end{cases}
$$

and $\bar{p}=\max _{1 \leq k \leq K, i \in \mathscr{G}_{k}} p_{k i}$

Proof. Let $\tilde{x}_{i}=x_{i}-x_{\theta_{k}}$ for $i \in \mathscr{G}_{k}, 1 \leq k \leq K$, and $\tilde{y}_{i}=\sum_{j \in \mathscr{G}_{k} \cup \theta_{k}} a_{i j}\left(x_{j}-x_{i}\right)+\sum_{k^{\prime} \neq k} \sum_{j \in \mathscr{G}_{k^{\prime}}} a_{i j} x_{j}$ for $i=1, \ldots, N$. Denote $\tilde{x}=\left(\tilde{x}_{1}, \ldots, \tilde{x}_{N}\right)^{\mathrm{T}}$ and $\tilde{y}=\left(\tilde{y}_{1}, \ldots, \tilde{y}_{N}\right)^{\mathrm{T}}$. Therefore, we obtain

$$
\tilde{y}=-H \tilde{x}=\left(\operatorname{diag}\left(-H_{1}, \ldots,-H_{K}\right)+\widetilde{A}\right) \tilde{x},
$$

where $H_{1}, \ldots, H_{K}$ are defined in (3) and

$$
\widetilde{A}=\left(\begin{array}{cccc}
0 & A_{12} & \cdots & A_{1_{K}} \\
A_{21} & 0 & \cdots & A_{2 K} \\
\vdots & \vdots & \ddots & \vdots \\
A_{K 1} & A_{K 2} & \cdots & 0
\end{array}\right) \in \mathbb{R}^{N \times N} .
$$

Here, each block $A_{k k^{\prime}}=\left(a_{i j}\right) \in \mathbb{R}^{\left|\mathscr{G}_{k}\right| \times\left|\mathscr{G}_{k^{\prime}}\right|}$ (inheriting from the weighted adjacency matrix $A$ ) characterizes the weights between agents in subgroups $\mathscr{G}_{k}$ and $\mathscr{G}_{k^{\prime}}$ for $k^{\prime} \neq k$. It follows from Assumption 4 that $H$ is strictly diagonally dominant and hence invertible [40]. Therefore, $\widetilde{x}=0$ if and only if $\tilde{y}=0$, meaning that the leader-follower fixed-time group consensus is achieved if only if $\tilde{y}$ converges to zero in fixed time.

In view of (6) and (10), we have $\dot{\tilde{y}}_{i}=\sum_{j \in \mathscr{G}_{k}} a_{i j}\left(u_{j}-\right.$ $\left.u_{i}\right)+a_{i \theta_{k}}\left(u_{\theta_{k}}-u_{i}\right)+\sum_{k^{\prime} \neq k} \sum_{j \in \mathscr{G}_{k^{\prime}}} a_{i j} u_{j}$. Consider the Lyapunov function

$$
\mathrm{V}(t)=\sum_{k=1}^{K} \sum_{i \in \mathscr{G}_{k}} p_{k i}\left(\frac{\alpha\left|\tilde{y}_{i}\right|^{p+1}}{p+1}+\frac{\beta\left|\widetilde{y}_{i}\right|^{q+1}}{q+1}\right)
$$

For each $i \in \mathscr{G}_{k}, 1 \leq k \leq K$, we write $u_{i}=u_{i 1}+u_{i 2}$, where

$$
\begin{aligned}
u_{i 1}= & \alpha\left\lfloor\sum_{j \in \mathscr{G}_{k} \cup \theta_{k}} a_{i j}\left(x_{j}-x_{i}\right)+\sum_{k^{\prime} \neq k} \sum_{j \in \mathscr{G}_{k^{\prime}}} a_{i j} x_{j}\right\rfloor^{p} \\
& +\beta\left\lfloor\sum_{j \in \mathscr{G}_{k} \cup \theta_{k}} a_{i j}\left(x_{j}-x_{i}\right)+\sum_{k^{\prime} \neq k} \sum_{j \in \mathscr{G}_{k^{\prime}}} a_{i j} x_{j}\right]^{q}
\end{aligned}
$$

and $u_{i 2}=\gamma \operatorname{sgn}\left(\sum_{j \in \mathscr{G}_{k} \cup \theta_{k}} a_{i j}\left(x_{j}-x_{i}\right)+\sum_{k^{\prime} \neq k} \sum_{j \in \mathscr{G}_{k^{\prime}}} a_{i j} x_{j}\right)$. The time derivative of (12) along the solution of (6) satisfies 


$$
\begin{aligned}
\dot{\mathrm{V}}(t)= & \sum_{k=1}^{K} \sum_{i \in \mathscr{G}_{k}}\left[p _ { k i } \left(\alpha\left\lfloor\left.\tilde{y}_{i}\right|^{p}+\beta\left\lfloor\left.\tilde{y}_{i}\right|^{q}\right) \cdot\left(\sum_{j \in \mathscr{G}_{k}} a_{i j}\left(u_{j}-u_{i}\right)+a_{i \theta_{k}}\left(u_{\theta_{k}}-u_{i}\right)+\sum_{k^{\prime} \neq k} \sum_{j \in \mathscr{G}_{k^{\prime}}} a_{i j} u_{j}\right)\right]\right.\right. \\
= & \sum_{k=1}^{K} \sum_{i \in \mathscr{G}_{k}}\left[p_{k i}\left(\alpha\left\lfloor\left.\tilde{y}_{i}\right|^{p}+\beta\left\lfloor\tilde{y}_{i}\right]^{q}\right) \cdot\left(\sum_{j \in \mathscr{G}_{k}} a_{i j}\left(u_{j 1}-u_{i 1}\right)-a_{i \theta_{k}} u_{i 1}+\sum_{k^{\prime} \neq k} \sum_{j \in \mathscr{G}_{k^{\prime}}} a_{i j} u_{j 1}\right)\right]\right. \\
& +\sum_{k=1}^{K} \sum_{i \in \mathscr{G}_{k}}\left[p _ { k i } \left(\alpha\left\lfloor\left.\tilde{y}_{i}\right|^{p}+\beta\left\lfloor\left.\tilde{y}_{i}\right|^{q}\right) \cdot\left(\sum_{j \in \mathscr{G}_{k}} a_{i j}\left(u_{j 2}-u_{i 2}\right)+a_{i \theta_{k}}\left(u_{\theta_{k}}-u_{i 2}\right)+\sum_{k^{\prime} \neq k} \sum_{j \in \mathscr{G}_{k^{\prime}}} a_{i j}\left(u_{j 2}-u_{i 2}\right)\right)\right],\right.\right.
\end{aligned}
$$

where we exploited Assumption 4 in the last term on the righthand side of (14). By observing that $u_{j 2}-u_{i 2} \leq \gamma-$ $\gamma \operatorname{sgn}\left(\tilde{y}_{i}\right), u_{\theta_{k}}-u_{i 2} \leq \omega-\gamma \operatorname{sgn}\left(\tilde{y}_{i}\right) \leq \gamma-\gamma \operatorname{sgn}\left(\tilde{y}_{i}\right)$, and $u_{j 2}-u_{i 2} \leq \gamma-\gamma \operatorname{sgn}\left(\widetilde{y}_{i}\right)$, we obtain from (14) that

$$
\begin{aligned}
& \dot{\mathrm{V}}(t) \leq \sum_{k=1}^{K} \sum_{i \in \mathscr{G}_{k}}\left[p_{k i}\left(\alpha\left\lfloor\tilde{y}_{i}\right\rceil^{p}+\beta\left\lfloor\tilde{y}_{i}\right]^{q}\right)\right. \\
&\left.\cdot\left(\sum_{j \in \mathscr{G}_{k}} a_{i j}\left(u_{j 1}-u_{i 1}\right)-a_{i \theta_{k}} u_{i 1}+\sum_{k^{\prime} \neq k} \sum_{j \in \mathscr{G}_{k^{\prime}}} a_{i j} u_{j 1}\right)\right] \\
&=-\frac{1}{2}\left(\alpha\lfloor\tilde{y}\rfloor^{p}+\beta\lfloor\tilde{y}\rfloor^{q}\right)^{\mathrm{T}} Q\left(\alpha\lfloor\tilde{y}\rfloor^{p}+\beta\lfloor\tilde{y}]^{q}\right) \\
&+\frac{1}{2}\left(\alpha\lfloor\tilde{y}\rfloor^{p}+\beta\lfloor\tilde{y}]^{q}\right)^{\mathrm{T}}\left[\operatorname{diag}\left(P_{1}, \ldots, P_{K}\right) \widetilde{A}\right. \\
&\left.+\widetilde{A}^{\mathrm{T}} \operatorname{diag}\left(P_{1}, \ldots, P_{K}\right)\right] \cdot\left(\alpha\lfloor\tilde{y}\rfloor^{p}+\beta\lfloor\tilde{y}]^{q}\right) .
\end{aligned}
$$

Recall the definition of $\rho$ in Assumption 9, and it follows from the Geršgorin disc theorem [40] that the eigenvalues of the matrix $\operatorname{diag}\left(P_{1}, \ldots, P_{K}\right) \widetilde{A}+\widetilde{A}^{\mathrm{T}} \operatorname{diag}\left(P_{1}, \ldots, P_{K}\right)$ lie within the interval $[-\rho, \rho]$. Hence, we have

$$
\begin{aligned}
\dot{\mathrm{V}}(t) \leq & -\frac{1}{2}\left(\lambda_{\min }(Q)-\rho\right)\left(\alpha\left\lfloor\left.\tilde{y}\right|^{p}+\beta\lfloor\tilde{y}]^{q}\right)^{\mathrm{T}}\right. \\
& \cdot\left(\alpha\left\lfloor\left.\tilde{y}\right|^{p}+\beta\lfloor\tilde{y}]^{q}\right)\right.
\end{aligned}
$$

by using the Rayleigh-Ritz theorem [40].

Next, we will estimate the righthand side of (16). Following [32], we set $h_{1}(\tilde{y})=\left(\alpha\lfloor\tilde{y}]^{p}+\beta\left\lfloor\left.\tilde{y}\right|^{q}\right)^{\mathrm{T}}\left(\alpha\lfloor\tilde{y}]^{p}+\right.\right.$ $\left.\beta\lfloor\tilde{y}]^{q}\right)=\alpha^{2} \sum_{i=1}^{N}\left|\tilde{y}_{i}\right|^{2 p}+2 \alpha \beta \sum_{i=1}^{N}\left|\tilde{y}_{i}\right|^{p+q}+\beta^{2} \sum_{i=1}^{N}\left|\tilde{y}_{i}\right|^{2 q}$, $h_{2}(\tilde{y})=\mathrm{V}^{2 p /(p+1)}(t)$, and $h_{3}(\tilde{y})=\mathrm{V}^{2 q /(q+1)}(t)$. It follows from (12) and Lemma 1 that

$$
\begin{aligned}
h_{2}(\tilde{y}) & \leq\left(\frac{\bar{p} \alpha}{p+1}\left(\sum_{i=1}^{N}\left|\tilde{y}_{i}\right|\right)^{p+1}\right. \\
+ & \left.\frac{\bar{p} \beta}{q+1}\left(\sum_{i=1}^{N}\left|\tilde{y}_{i}\right|\right)^{q+1}\right)^{2 p /(p+1)} \leq\left(\frac{\bar{p} \alpha}{p+1}\right)^{2 p /(p+1)}
\end{aligned}
$$

$$
\begin{aligned}
& \cdot\left(\sum_{i=1}^{N}\left|\widetilde{y}_{i}\right|\right)^{2 p}+\left(\frac{\bar{p} \beta}{q+1}\right)^{2 p /(p+1)} \\
& \cdot\left(\sum_{i=1}^{N}\left|\widetilde{y}_{i}\right|\right)^{2 p(q+1) /(p+1)}
\end{aligned}
$$

since $p, q>0$ and $0<2 p /(p+1)<1$. Similarly, since $2 q /(q+$ 1) $>1$, we obtain

$$
\begin{aligned}
& h_{3}(\widetilde{y}) \leq\left(\frac{\bar{p} \alpha}{p+1}\left(\sum_{i=1}^{N}\left|\widetilde{y}_{i}\right|\right)^{p+1}\right. \\
& \left.+\frac{\bar{p} \beta}{q+1}\left(\sum_{i=1}^{N}\left|\widetilde{y}_{i}\right|\right)^{q+1}\right)^{2 q /(q+1)} \\
& \quad \leq 2^{(q-1) /(q+1)}\left(\frac{\bar{p} \alpha}{p+1}\right)^{2 q /(q+1)}\left(\sum_{i=1}^{N}\left|\widetilde{y}_{i}\right|\right)^{2 q(p+1) /(q+1)} \\
& +2^{(q-1) /(q+1)}\left(\frac{\bar{p} \beta}{q+1}\right)^{2 q /(q+1)}\left(\sum_{i=1}^{N}\left|\tilde{y}_{i}\right|\right)^{2 q} .
\end{aligned}
$$

We consider two cases according to the value of $p$.

Case $1(0<p \leq 1 / 2)$. In this case, we have

$$
\begin{aligned}
h_{1}(\tilde{y}) \geq & \alpha^{2}\left(\sum_{i=1}^{N}\left|\widetilde{y}_{i}\right|\right)^{2 p}+2 \alpha \beta N^{1-p-q}\left(\sum_{i=1}^{N}\left|\widetilde{y}_{i}\right|\right)^{p+q} \\
& +\beta^{2} N^{1-2 q}\left(\sum_{i=1}^{N}\left|\widetilde{y}_{i}\right|\right)^{2 q}
\end{aligned}
$$

by Lemma 1. If $\sum_{i=1}^{N}\left|\tilde{y}_{i}\right| \geq 1$, then $h_{1}(\tilde{y}) \geq$ $\beta^{2} N^{1-2 q}\left(\sum_{i=1}^{N}\left|\tilde{y}_{i}\right|\right)^{2 q}$ by (19), and moreover, $\left(\sum_{i=1}^{N}\left|\tilde{y}_{i}\right|\right)^{2 p} \leq$ $\left(\sum_{i=1}^{N}\left|\tilde{y}_{i}\right|\right)^{2 q},\left(\sum_{i=1}^{N}\left|\tilde{y}_{i}\right|\right)^{2 p(q+1) /(p+1)} \leq\left(\sum_{i=1}^{N}\left|\tilde{y}_{i}\right|\right)^{2 q}$, and $\left(\sum_{i=1}^{N}\left|\tilde{y}_{i}\right|\right)^{2 q(p+1) /(q+1)} \leq\left(\sum_{i=1}^{N}\left|\tilde{y}_{i}\right|\right)^{2 q}$. Recalling the definition of $c_{1}$, we have

$$
h_{1}(\tilde{y}) \geq \frac{\beta^{2} N^{1-2 q}}{c_{1}}\left(h_{2}(\tilde{y})+h_{3}(\tilde{y})\right)
$$


by using (17) and (18). If $\sum_{i=1}^{N}\left|\tilde{y}_{i}\right| \leq 1$, then $h_{1}(\widetilde{y}) \geq$ $\alpha^{2}\left(\sum_{i=1}^{N}\left|\widetilde{y}_{i}\right|\right)^{2 p}$ by (19), and moreover, $\left(\sum_{i=1}^{N}\left|\tilde{y}_{i}\right|\right)^{2 q} \leq$ $\left(\sum_{i=1}^{N}\left|\tilde{y}_{i}\right|\right)^{2 p},\left(\sum_{i=1}^{N}\left|\tilde{y}_{i}\right|\right)^{2 p(q+1) /(p+1)} \leq\left(\sum_{i=1}^{N}\left|\tilde{y}_{i}\right|\right)^{2 p}$, and $\left(\sum_{i=1}^{N}\left|\tilde{y}_{i}\right|\right)^{2 q(p+1) /(q+1)} \leq\left(\sum_{i=1}^{N}\left|\widetilde{y}_{i}\right|\right)^{2 p}$. Analogously, it follows from (17) and (18) that

$$
h_{1}(\tilde{y}) \geq \frac{\alpha^{2}}{c_{1}}\left(h_{2}(\tilde{y})+h_{3}(\tilde{y})\right) .
$$

Case $2(1 / 2 \leq p<1)$. In this case, we have

$$
\begin{aligned}
h_{1}(\widetilde{y}) \geq & \alpha^{2} N^{1-2 p}\left(\sum_{i=1}^{N}\left|\tilde{y}_{i}\right|\right)^{2 p} \\
& +2 \alpha \beta N^{1-p-q}\left(\sum_{i=1}^{N}\left|\tilde{y}_{i}\right|\right)^{p+q} \\
& +\beta^{2} N^{1-2 q}\left(\sum_{i=1}^{N}\left|\tilde{y}_{i}\right|\right)^{2 q}
\end{aligned}
$$

by Lemma 1. If $\sum_{i=1}^{N}\left|\widetilde{y}_{i}\right| \geq 1$, we recover (20) similarly. If $\sum_{i=1}^{N}\left|\tilde{y}_{i}\right| \leq 1$, it follows again from (17) and (18) that

$$
h_{1}(\tilde{y}) \geq \frac{\alpha^{2} N^{1-2 p}}{c_{1}}\left(h_{2}(\tilde{y})+h_{3}(\tilde{y})\right) .
$$

Combining (20), (21), and (23) with (16), we are led to the conclusion that

$$
\begin{aligned}
\dot{\mathrm{V}}(t) & \leq-\frac{c_{2}}{2 c_{1}}\left(\lambda_{\text {min }}(Q)-\rho\right)\left(h_{2}(\widetilde{y})+h_{3}(\tilde{y})\right) \\
& \leq-\frac{c_{2}}{2 c_{1}}\left(\lambda_{\min }(Q)-\rho\right)\left(\mathrm{V}^{2 p /(p+1)}(t)+\mathrm{V}^{2 q /(q+1)}(t)\right) .
\end{aligned}
$$

Recall that $q=1 / p$, and Lemma 7 yields that the convergence time is bounded by $T_{\max }=c_{1} \pi(1+p) / c_{2}\left(\lambda_{\min }(Q)-\rho\right)(1-$ $p$ ) regardless of initial conditions by taking $a=b=$ $\left(c_{2} / 2 c_{1}\right)\left(\lambda_{\min }(Q)-\rho\right)$ and $\mu=(1+p) / 2(1-p)>1 / 2$. Based on the comments above, the leader-follower fixed-time group consensus is achieved and the proof is complete.

Remark 11. Notice that the convergence time upper bound $T_{\max }$ is fixed regardless of the initial conditions of the agents. Moreover, it is easy to check that $T_{\max }$ decreases with respect to $\alpha$ and $\beta$, but independent of $\gamma$. When $K=1$, that is, there exists only one leader, the problem reduces to the fixed-time leader-follower consensus, which has been solved in [32].

Remark 12. It is worth noting that the fixed-time group consensus tracking method studied in [27] essentially relies on the symmetry of the communication topology of each subgroup, which is not applicable here. Without the symmetry condition, a more careful analysis with two cases regarding the range of parameter $p$ is needed. The parameter $0<$ $p<1$ contributes to the fixed-time stability $[23,24]$, and the division of the two cases is due to technical reasons. Note that the dependence of upper bound of the settling time, $T_{\max }$, on $p$ is involved and nonmonotone (recall that $q=1 / p$ ). Thus, the convergence time for the two cases $p<1 / 2$ and $p>1 / 2$ is not comparable in general.

Remark 13. In controller (7), the third term $\gamma \operatorname{sgn}\left(\sum_{j \in \mathscr{G}_{k} \cup \theta_{k}} a_{i j}\left(x_{j}-x_{i}\right)+\sum_{k^{\prime} \neq k} \sum_{j \in \mathscr{G}_{k^{\prime}}} a_{i j} x_{j}\right)$ aims to deal with the dynamic leaders. If $\omega=0$, that is, the leaders are static, one can choose the parameter $\gamma=0$. In this case, the control protocol becomes

$$
\begin{aligned}
u_{i}= & \alpha\left\lfloor\sum_{j \in \mathscr{G}_{k} \cup \theta_{k}} a_{i j}\left(x_{j}-x_{i}\right)+\sum_{k^{\prime} \neq k} \sum_{j \in \mathscr{G}_{k^{\prime}}} a_{i j} x_{j}\right\rfloor^{p} \\
& +\beta\left\lfloor\sum_{j \in \mathscr{G}_{k} \cup \theta_{k}} a_{i j}\left(x_{j}-x_{i}\right)+\sum_{k^{\prime} \neq k} \sum_{j \in \mathscr{G}_{k^{\prime}}} a_{i j} x_{j}\right\rfloor^{q}
\end{aligned}
$$

for $i \in \mathscr{G}_{k}, k=1, \ldots, K$, which is consistent with the protocol in [24] in the special case of $K=1$ and $A=A^{\mathrm{T}}$.

\section{Numerical Examples}

In this section, we present some numerical examples to illustrate our theoretical results. Consider a multiagent system (6)-(7) with $K=3$ leader agents and $N=9$ follower agents having $\mathscr{G}_{1}=\{1,2,3,4\}, \mathscr{G}_{2}=\{5,6\}$, and $\mathscr{G}_{3}=\{7,8,9\}$. The network topology $G$ together with its associated edge weights is shown in Figure 1. The control inputs for the three leaders are taken as $u_{\theta_{1}}=-1, u_{\theta_{2}}=1+\cos (t)$, and $u_{\theta_{3}}=2 \cos (t)$; they are bounded by $\omega=2$. By direct calculations, we have $\rho=1.2$ and $\lambda_{\min }(Q)=1.5$. It is then easy to check that Assumptions 3, 4, and 9 are true. It is noteworthy that some negative weights between different subgroups are present. They indicate that the interactions between nodes in different subgroup can be competitive $[27,36]$, which play a crucial role in the analysis of bipartite consensus over signed graphs [41].

Example $1(p=1 / 3$ and $q=3)$. In this example, we consider the case of $p=1 / 3<1 / 2$. By taking $\alpha=\beta=1$ and $\gamma=2$, we obtain from Theorem 10 an explicit estimation of the settling time as $T_{\max } \approx 4.5 \times 10^{6} \mathrm{~s}$, which is independent of the initial conditions of the multiagent system. With the initial conditions $\left(x_{\theta_{1}}(0), x_{\theta_{2}}(0), x_{\theta_{3}}(0), x_{1}(0), x_{2}(0), x_{3}(0), x_{4}(0), x_{5}(0)\right.$, $\left.x_{6}(0), x_{7}(0), x_{8}(0), x_{9}(0)\right)=(-3,1,6,-2,4,-1,3,4,-6,4,3$, $-5)$ and $\left(x_{\theta_{1}}(0), x_{\theta_{2}}(0), x_{\theta_{3}}(0), x_{1}(0), x_{2}(0), x_{3}(0), x_{4}(0), x_{5}(0)\right.$, $\left.x_{6}(0), x_{7}(0), x_{8}(0), x_{9}(0)\right)=(150,100,-200,-130,200,-150$, $300,250,-125,300,-250,-100)$, the simulation results are shown in Figures 2(a) and 2(b), respectively. We observe that, for all the initial conditions, the follower agents are able to track their corresponding leaders quite rapidly. In view of the conservativeness of our theoretical estimation, a more practical settling time could be derived by simulating the dynamical system for the followers with sufficiently large initial conditions. This is viable since the fixed-time convergence is theoretically guaranteed and thus the convergence time will tend to a finite limit as the initial conditions increase. As 


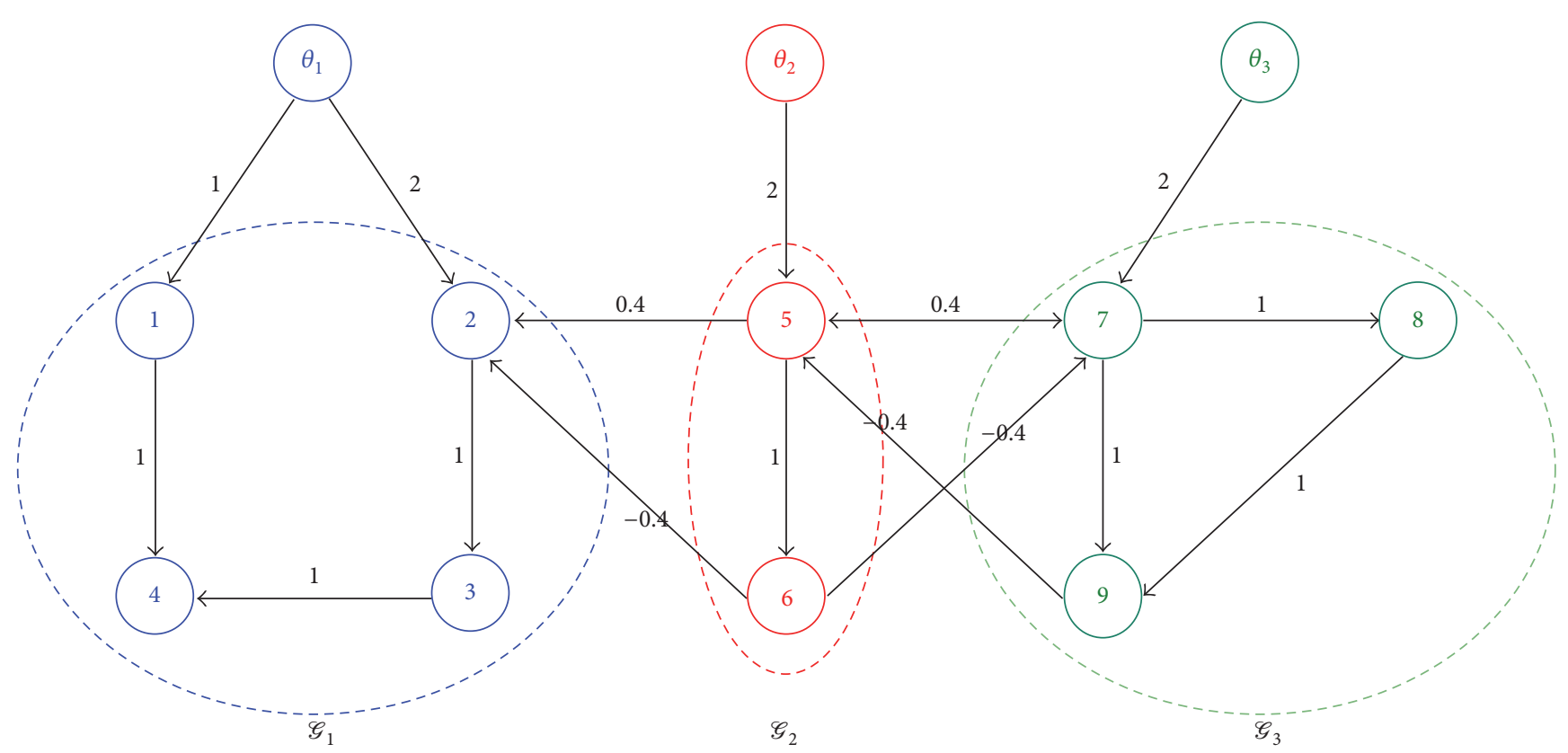

FIgURE 1: Communication topology.

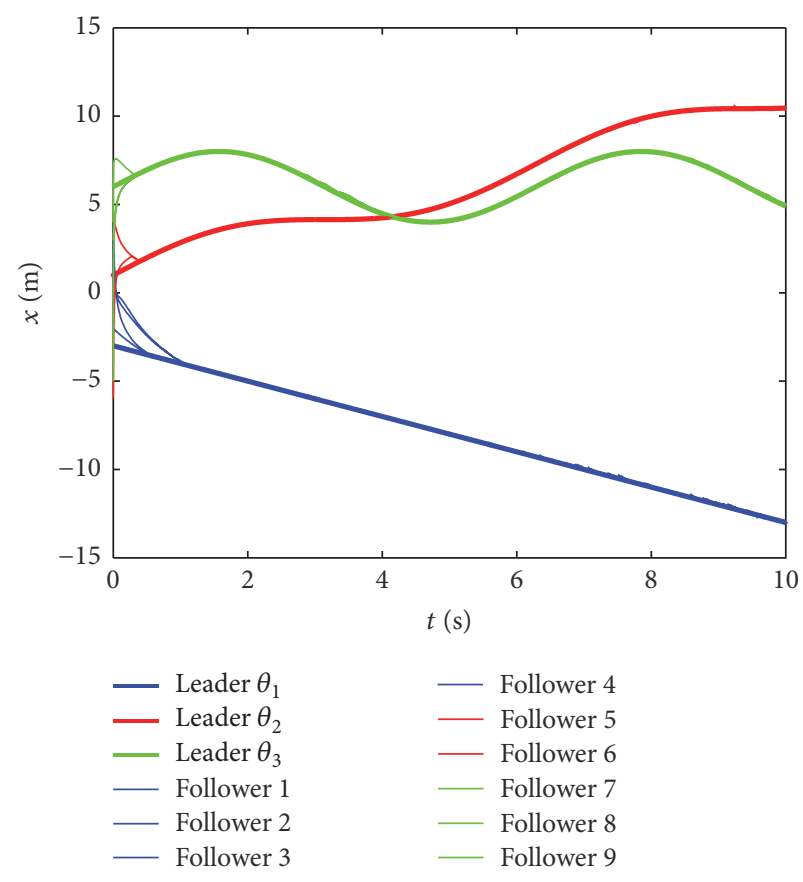

(a)

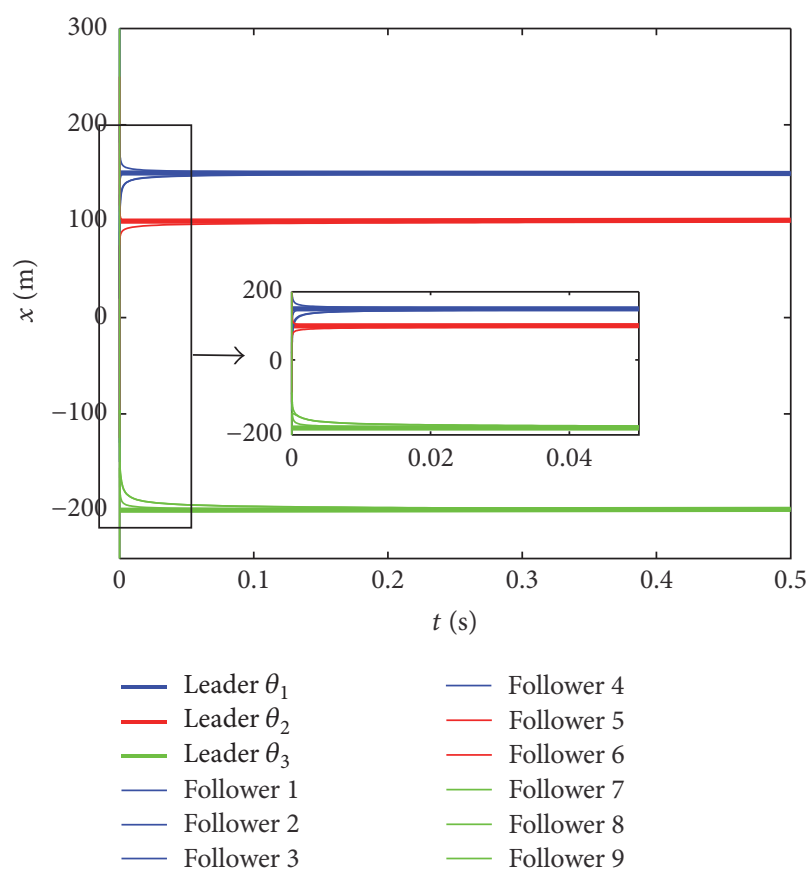

(b)

FIGURE 2: Leader-follower fixed-time group consensus for the multiagent system (6)-(7) in Example 1. (a) is for $\left(x_{\theta_{1}}(0), x_{\theta_{2}}(0)\right.$, $\left.x_{\theta_{3}}(0), x_{1}(0), x_{2}(0), x_{3}(0), x_{4}(0), x_{5}(0), x_{6}(0), x_{7}(0), x_{8}(0), x_{9}(0)\right)=(-3,1,6,-2,4,-1,3,4,-6,4,3,-5)$; and $(\mathrm{b})$ is for $\left(x_{\theta_{1}}(0), x_{\theta_{2}}(0), x_{\theta_{3}}(0)\right.$, $\left.x_{1}(0), x_{2}(0), x_{3}(0), x_{4}(0), x_{5}(0), x_{6}(0), x_{7}(0), x_{8}(0), x_{9}(0)\right)=(150,100,-200,-130,200,-150,300,250,-125,300,-250,-100)$.

such, we estimate the convergence time as about $10 \mathrm{~s}$ through simulations over sufficiently large initial conditions.

Example $2(p=2 / 3$ and $q=3 / 2)$. In this example, we consider the case of $p=2 / 3>1 / 2$. By taking $\alpha=$ $\beta=1$ and $\gamma=2$ again, we obtain from Theorem 10 an explicit estimation of the settling time as $T_{\max } \approx 1.3 \times$ $10^{4} \mathrm{~s}$, which is independent of the initial conditions of the multiagent system. With the same initial conditions as in Example 1, the simulation results are shown in Figures 3(a) and 3(b), respectively. Similarly, the estimated settling time upper bound is very conservative. By simulating the system 


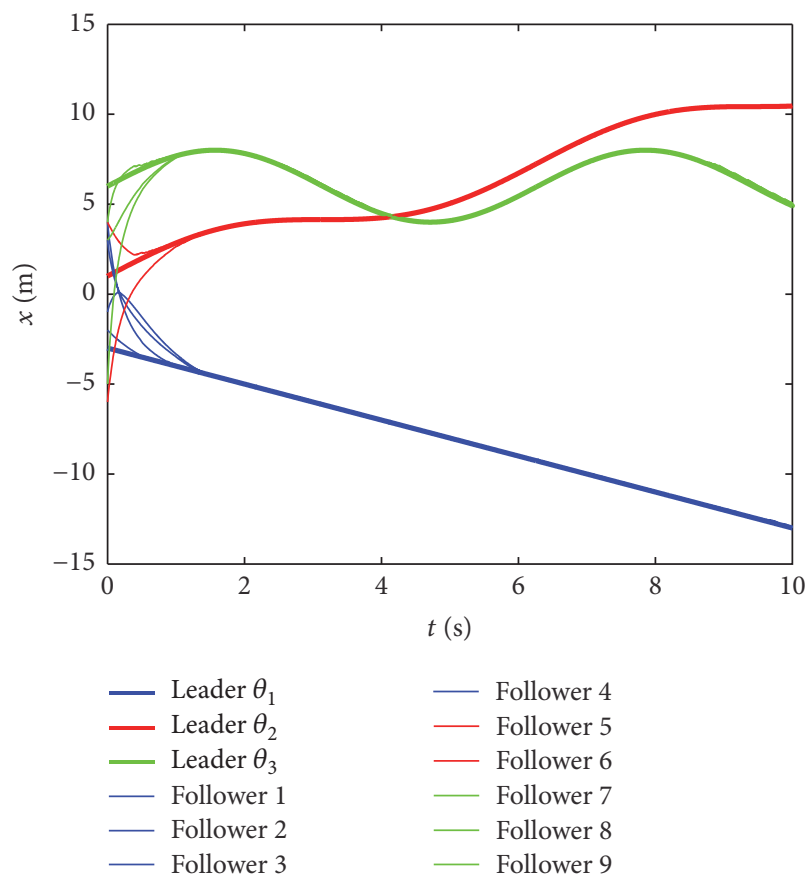

(a)

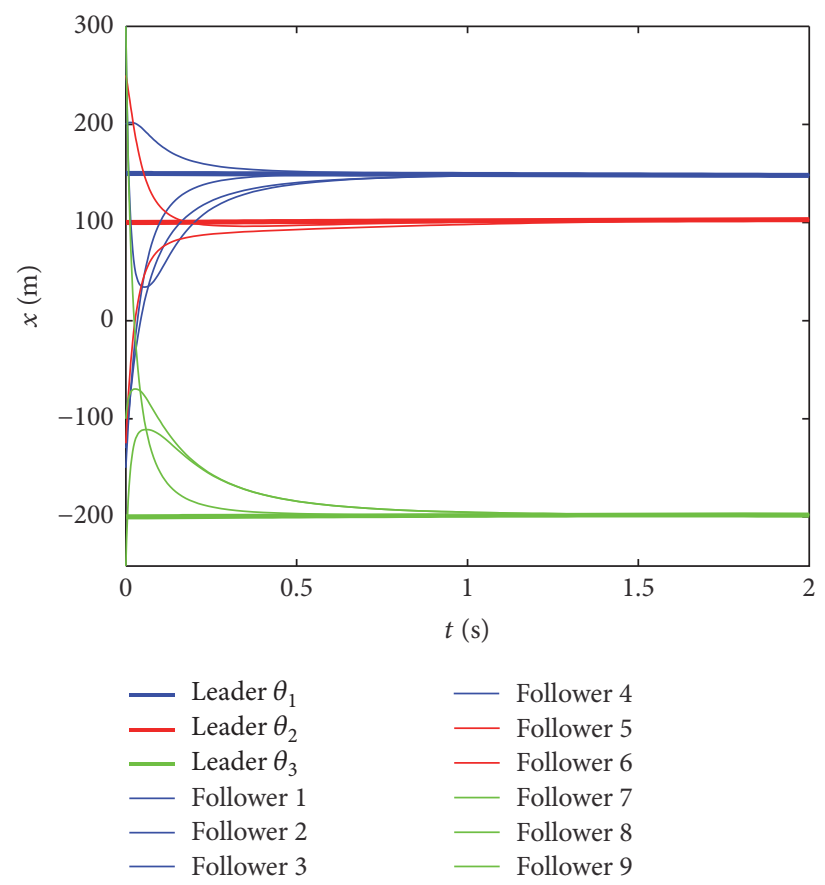

(b)

FIGURE 3: Leader-follower fixed-time group consensus for the multiagent system (6)-(7) in Example 2. (a) is for $\left(x_{\theta_{1}}(0), x_{\theta_{2}}(0), x_{\theta_{3}}(0)\right.$, $\left.x_{1}(0), x_{2}(0), x_{3}(0), x_{4}(0), x_{5}(0), x_{6}(0), x_{7}(0), x_{8}(0), x_{9}(0)\right)=(-3,1,6,-2,4,-1,3,4,-6,4,3,-5)$; and (b) is for $\left(x_{\theta_{1}}(0), x_{\theta_{2}}(0), x_{\theta_{3}}(0), x_{1}(0)\right.$, $\left.x_{2}(0), x_{3}(0), x_{4}(0), x_{5}(0), x_{6}(0), x_{7}(0), x_{8}(0), x_{9}(0)\right)=(150,100,-200,-130,200,-150,300,250,-125,300,-250,-100)$.

with sufficiently large initial conditions, the convergence time can be determined as about $10 \mathrm{~s}$.

\section{Conclusions}

In this paper, the fixed-time consensus problem is generalized to the group tracking control of multiagent systems with multiple leaders and the underlying communication topology is composed of a directed network. A new distributed controller is proposed which solves the leader-follower fixed-time group consensus problem. An explicit estimation of the settling time is provided regardless of the initial conditions. As future works, we will consider how to obtain sharper estimation of the settling time over directed networks and study the effect of communication delays as well as robustness against disturbances.

\section{Conflicts of Interest}

The authors declare that there are no conflicts of interest regarding the publication of this paper.

\section{Acknowledgments}

This work was funded by the National Natural Science Foundation of China (11505127) and the Shanghai Pujiang Program (15PJ1408300).

\section{References}

[1] A. Jadbabaie, J. Lin, and A. S. Morse, "Coordination of groups of mobile autonomous agents using nearest neighbor rules," IEEE Transactions on Automatic Control, vol. 48, no. 6, pp. 988-1001, 2003.

[2] R. Olfati-Saber and R. M. Murray, "Consensus problems in networks of agents with switching topology and time-delays," IEEE Transactions on Automatic Control, vol. 49, no. 9, pp. 15201533, 2004.

[3] W. Ren and R. W. Beard, "Consensus seeking in multiagent systems under dynamically changing interaction topologies," IEEE Transactions on Automatic Control, vol. 50, no. 5, pp. 655661, 2005.

[4] R. Olfati-Saber, J. A. Fax, and R. M. Murray, "Consensus and cooperation in networked multi-agent systems," Proceedings of the IEEE, vol. 95, no. 1, pp. 215-233, 2007.

[5] Y. Cao, W. Yu, W. Ren, and G. Chen, "An overview of recent progress in the study of distributed multi-agent coordination," IEEE Transactions on Industrial Informatics, vol. 9, no. 1, pp. 427-438, 2013.

[6] J. Yu and L. Wang, "Group consensus in multi-agent systems with switching topologies and communication delays," Systems \& Control Letters, vol. 59, no. 6, pp. 340-348, 2010.

[7] J. Yu and L. Wang, "Group consensus of multi-agent systems with directed information exchange," International Journal of Systems Science, vol. 43, no. 2, pp. 334-348, 2012.

[8] Y. Han, W. Lu, and T. Chen, "Achieving cluster consensus in continuous-time networks of multi-agents with inter-cluster non-identical inputs," IEEE Transactions on Automatic Control, vol. 60, no. 3, pp. 793-798, 2015. 
[9] Y. Wang, Z. Ma, J. Cao, A. Alsaedi, and F. E. Alsaadi, "Adaptive cluster synchronization in directed networks with nonidentical nonlinear dynamics," Complexity, vol. 21, no. S2, pp. 380-387, 2016.

[10] Y. Shang, " $L^{1}$ group consensus of multi-agent systems with switching topologies and stochastic inputs," Physics Letters. A, vol. 377, no. 25-27, pp. 1582-1586, 2013.

[11] Y. Shang, "A combinatorial necessary and sufficient condition for cluster consensus," Neurocomputing, vol. 216, pp. 611-616, 2016.

[12] T. H. Lee, J. H. Park, D. H. Ji, and H. Y. Jung, "Leader-following consensus problem of heterogeneous multi-agent systems with nonlinear dynamics using fuzzy disturbance observer," Complexity, vol. 19, no. 4, pp. 20-31, 2014.

[13] Q. Cui, D. Xie, and F. Jiang, "Group consensus tracking control of second-order multi-agent systems with directed fixed topology," Neurocomputing, vol. 218, pp. 286-295, 2016.

[14] Z. Tu, D. Zhang, X. Xia, and H. Yu, "Event-triggered group consensus of leader-following multi-agent systems with nonlinear dynamics," in Proceedings of the 35th Chinese Control Conference (CCC '16), pp. 7885-7890, Chengdu, China, July 2016.

[15] S. P. Bhat and D. S. Bernstein, "Finite-time stability of continuous autonomous systems," SIAM Journal on Control and Optimization, vol. 38, no. 3, pp. 751-766, 2000.

[16] L. Wang and F. Xiao, "Finite-time consensus problems for networks of dynamic agents," IEEE Transactions on Automatic Control, vol. 55, no. 4, pp. 950-955, 2010.

[17] Y. Cao and W. Ren, "Finite-time consensus for multi-agent networks with unknown inherent nonlinear dynamics," Automatica, vol. 50, no. 10, pp. 2648-2656, 2014.

[18] X. Liu, J. Lam, W. Yu, and G. Chen, "Finite-time consensus of multiagent systems with a switching protocol," IEEE Transactions on Neural Networks and Learning Systems, vol. 27, no. 4, pp. 853-862, 2016.

[19] Y. Han, D. Zhao, and Y. Sun, "Finite-time flocking problem of a Cucker-smale-type self-propelled particle model," Complexity, vol. 21, pp. 354-361, 2016.

[20] G. Mei, X. Wu, D. Ning, and J.-A. Lu, "Finite-time stabilization of complex dynamical networks via optimal control," Complexity, vol. 21, pp. 417-425, 2016.

[21] W. Cui, J.-a. Fang, W. Zhang, and X. Wang, "Finite-time cluster synchronisation of Markovian switching complex networks with stochastic perturbations," IET Control Theory \& Applications, vol. 8, no. 1, pp. 30-41, 2014.

[22] Y. Shang, "Finite-time cluster average consensus for networks via distributed iterations," International Journal of Control, Automation and Systems, 2017.

[23] A. Polyakov, "Nonlinear feedback design for fixed-time stabilization of linear control systems," IEEE Transactions on Automatic Control, vol. 57, no. 8, pp. 2106-2110, 2012.

[24] S. Parsegov, A. Polyakov, and P. Shcherbakov, "Fixed-time consensus algorithm for multi-agent systems with integrator dynamics," in Proceedings of the 4th IFAC Workshop on Distributed Estimation and Control in Networked Systems, pp. 110115, Koblenz, Germany, September, 2013.

[25] Z. Zuo and L. Tie, "A new class of finite-time nonlinear consensus protocols for multi-agent systems," International Journal of Control, vol. 87, no. 2, pp. 363-370, 2014.

[26] Z. Zuo and L. Tie, "Distributed robust finite-time nonlinear consensus protocols for multi-agent systems," International Journal of Systems Science, vol. 47, no. 6, pp. 1366-1375, 2016.
[27] X. Liu and T. Chen, "Fixed-time cluster synchronization for complex networks via pinning control," https://arxiv.org/ abs/1509.03350.

[28] J. Fu and J. Wang, "Fixed-time coordinated tracking for secondorder multi-agent systems with bounded input uncertainties," Systems \& Control Letters, vol. 93, pp. 1-12, 2016.

[29] M. Defoort, A. Polyakov, G. Demesure, M. Djemai, and K. Veluvolu, "Leader-follower fixed-time consensus for multiagent systems with unknown non-linear inherent dynamics," IET Control Theory \& Applications, vol. 9, no. 14, pp. 2165-2170, 2015.

[30] Y. Wan, J. Cao, G. Wen, and W. Yu, "Robust fixed-time synchronization of delayed Cohen-Grossberg neural networks," Neural Networks, vol. 73, pp. 86-94, 2016.

[31] J. Cao and R. Li, "Fixed-time synchronization of delayed memristor-based recurrent neural networks," Science China Information Sciences, vol. 60, no. 3, Article ID 032201, 2017.

[32] J. Fu and J. Wang, "Finite-time consensus for multi-agent systems with globally bounded convergence time under directed communication graphs," International Journal of Control, 2016.

[33] G. H. Hardy, J. E. Littlewood, and G. Pólya, Inequalities, vol. 2nd, Cambridge, at the University Press, 1952.

[34] M. Mesbahi and M. Egerstedt, Graph Theoretic Methods in Multiagent Networks, Princeton University Press, New Jersey, NJ, USA, 2010.

[35] H. Zhang, Z. Li, Z. Qu, and F. L. Lewis, "On constructing Lyapunov functions for multi-agent systems," Automatica, vol. 58, pp. 39-42, 2015.

[36] Y. Shang, "Couple-group consensus of continuous-time multiagent systems under Markovian switching topologies," Journal of the Franklin Institute, vol. 352, no. 11, pp. 4826-4844, 2015.

[37] M. Nagy, Z. Ákos, D. Biro, and T. Vicsek, "Hierarchical group dynamics in pigeon flocks," Nature, vol. 464, no. 7290 , pp. $890-$ 893, 2010.

[38] Y. Shang and R. Bouffanais, "Influence of the number of topologically interacting neighbors on swarm dynamics," Scientific Reports, vol. 4, article 4184, 2014.

[39] A. F. Filippov, Differential Equations with Discontinuous RightHand Side, Kluwer Academic Publishers, Dordrecht, The Netherlands, 1988.

[40] R. A. Horn and C. R. Johnson, Matrix analysis, Cambridge University Press, New York, NY, USA, 1985.

[41] C. Altafini, "Consensus problems on networks with antagonistic interactions," IEEE Transactions on Automatic Control, vol. 58, no. 4, pp. 935-946, 2013. 


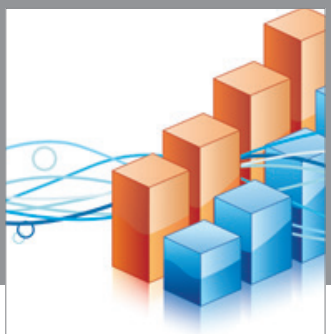

Advances in

Operations Research

vatem alat4

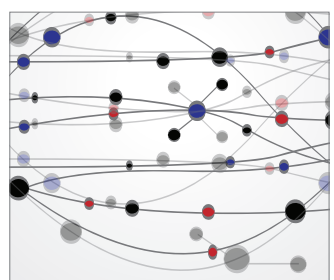

\section{The Scientific} World Journal
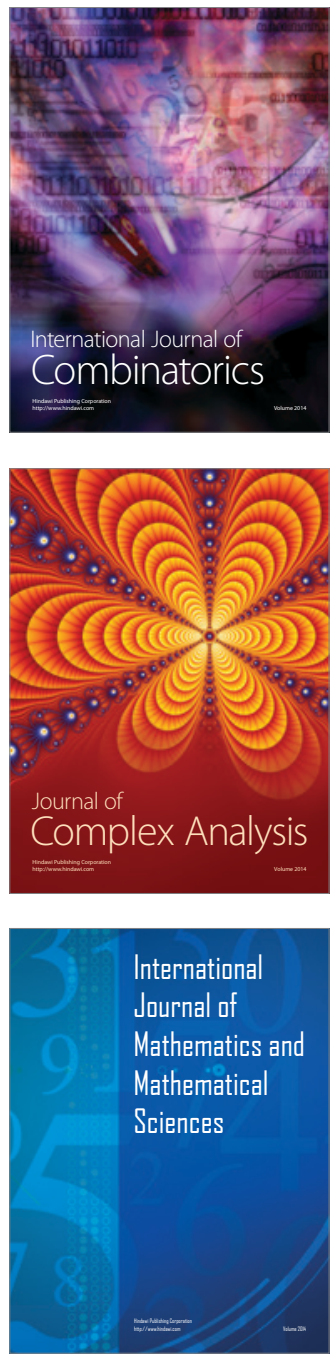
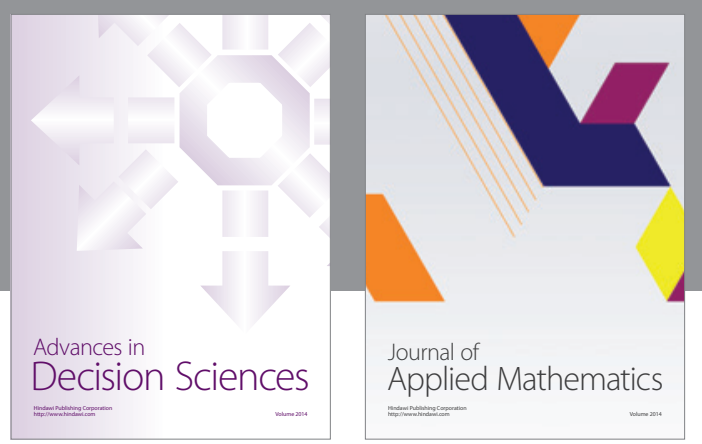

Algebra

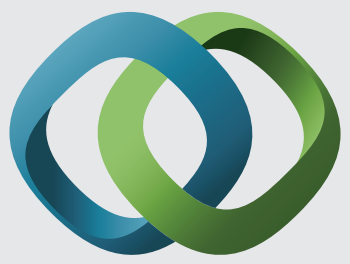

\section{Hindawi}

Submit your manuscripts at

https://www.hindawi.com
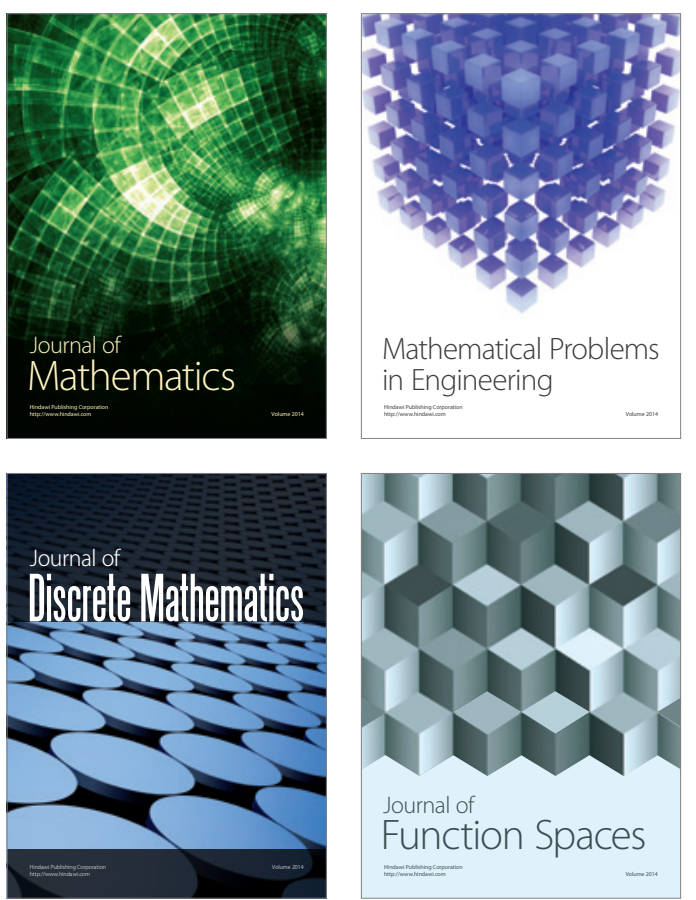

Mathematical Problems in Engineering
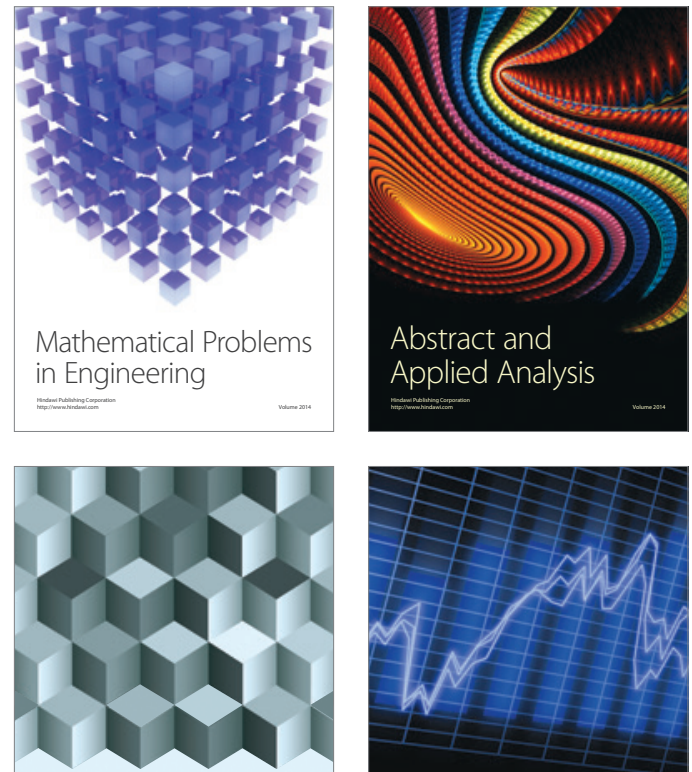

Journal of

Function Spaces

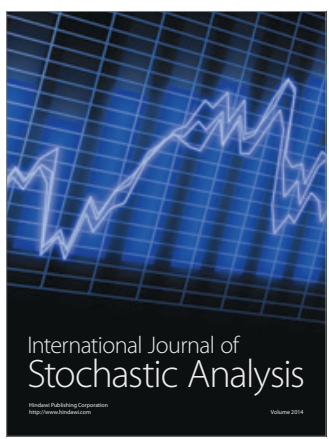

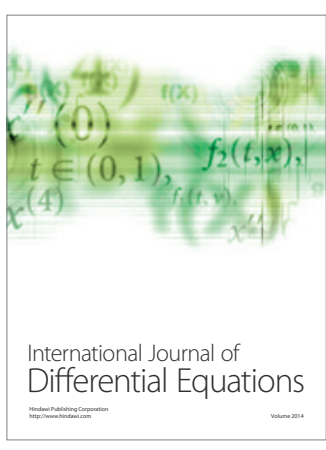
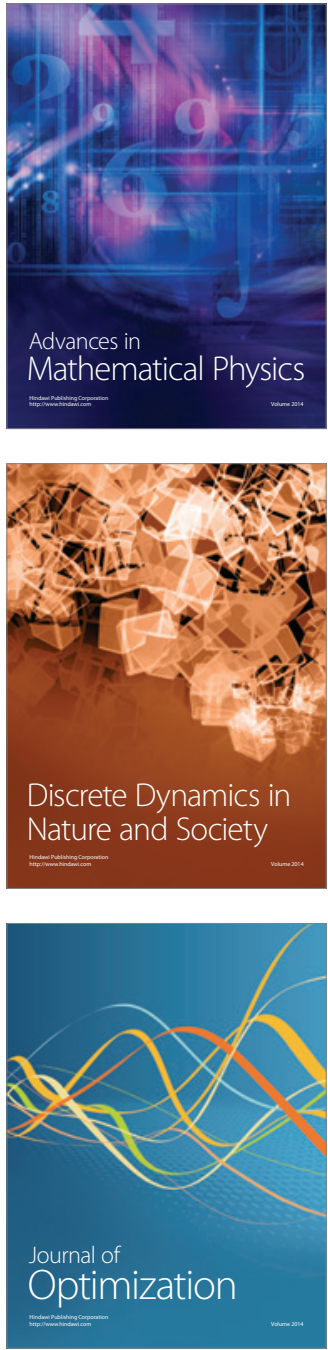